\title{
Multifunctional Magnetic Iron Oxide Nanoparticles for Intracellular Imaging and Drug Delivery to Cancer Cells
}

\author{
Kheireddine El-Boubbou
}

\begin{abstract}
Nowadays there is a tremendous shift in focus towards developing controlled drug delivery systems using drug-loaded nanomaterials. Of the many available systems, magnetic nanoparticles are considered promising drug-carriers due to their large surface area, ease of synthesis, low toxicity and imaging capability. Herein, we report a simple approach to create a novel chemotherapeutic formulation made of fluorescently-labeled PVPylated magnetic iron oxide nanoparticles loaded with anticancer drugs. Drug loading and release profiles were first studied, showing high loading drug efficiencies and relatively fast release of the cargo from its construct. The delivery of these particles to cancerous cells were then investigated. Whereas the Fl-PVP-MNPs were not toxic to the tested cancerous cells even at high doses, the cells were sensitive to Dox-loaded Fl-PVP-MNPs, effectively killing the cells. We anticipate that the observed potent effect is due to the successful intracellular uptake of the MNPs by cells and the subsequent release of Dox from the NPs, which can then translocate to the nucleus and exert its cytotoxic action. Notably, the MNPs used in this work can potentially open new opportunities for in vivo cancer therapeutic imaging and hyperthermia.
\end{abstract}

Index Terms-Magnetic nanoparticles, metal oxide, drug delivery, cancer cells.

\section{INTRODUCTION}

Drug delivery systems are receiving immense attention for pharmaceutical and biomedical applications due to their potential to improve targeting, enhance the therapeutic efficacy, and reduce the systemic side effects of drugs [1], [2]. Since tumors are believed to develop resistance to chemotherapy shortly after the first chemotherapeutic regimes, new approaches are required that can specifically target individual cancer cells in order to better control and reduce their progression [3]. Recently, innovative therapeutic approaches beyond the conventional therapies of using chemical drugs are being actively developed [4]. Using different types of novel nanoplatforms, researchers are demonstrating the use of functional nanomaterials to carry a pharmaceutical drug, direct its delivery, and enhance its local concentration, giving promise for next-generation disease treatment [5], [6]. Of the many varieties, magnetic metal oxide nanoparticles (MNPs), specifically made of iron oxides $\left(\mathrm{Fe}_{3} \mathrm{O}_{4}\right)$, have been widely popularized as plausible drug carriers because of their large surface areas, ease of syntheses

Manuscript received December 9, 2015; revised August 6, 2016

Kheireddine El-Boubbou is with King Saud bin Abdulaziz University for Health Sciences (KSAU-HS) and King Abdullah International Medical Research Center (KAIMRC), King Abdulaziz Medical City, National Guard Health Affairs, Riyadh 11481, Kingdom of Saudi Arabia (e-mail: elboubboukh@ngha.med.sa; boubbouk@ksau-hs.edu.sa). and surface functionalities [7]-[10]. Moreover, their superparamagnetism, imagability, low toxicity, biocompatibility, and tunable sizes, made such high-demand materials desirable for cancer theranostics [11]. The theranostics capability of MNPs along with their high biocompatibility and stability compared to other delivery systems, makes them more attractive candidates for drug delivery applications. Importantly, multiple types of agents including fluorophores and chemotherapeutic agents can be functionalized onto the MNPs. Co-assembling fluorescent and therapeutic drugs onto a magnetic construct, offers the possibility of producing new classes of super-nanoparticles with a set of combined potentials for in vivo photon and MR imaging.

In this work, a novel chemotherapeutic formulation made of poly- $N$-vinyl-pyrrolidone (PVP)-stabilized fluoresceinated MNPs of ferrites loaded with the anticancer drug Doxorubicin (Dox) was developed. In vitro release studies, toxicities, intracellular release properties, and abilities to kill cancerous cells were also evaluated.

\section{EXPERIMENTAL DETAILS}

Methods Fourier transform infrared (FTIR) spectra (400-4000 $\mathrm{cm}^{-1}$ ) were recorded as $\mathrm{KBr}$ pellets using Shimadzu IRAffinity-1. UV-vis measurements were conducted on Evolution 60S UV-vis Spectrophotometer. Dynamic light scattering (DLS) measurements were assessed on Malvern Zetasizer Nano ZS instrument. Transmission electron microscopy (TEM) images were collected on a JEOL-JEM 1230 operating at $100 \mathrm{kV}$ using Gatan camera with Digital Micrograph Imaging software. Confocal images were visualized using inverted Zeiss LSM 780 multiphoton laser scanning confocal microscopy.

Synthesis of Dox-loaded PVPylated MNPs. $1 \mathrm{~mL}$ aqueous dispersion of Fl-labeled PVPylated MNPs $(1.5 \mathrm{mg} / \mathrm{mL})$ and 1 $\mathrm{mL}$ of Dox.HCl solution $(500 \mu \mathrm{g} / \mathrm{mL})$ containing few drops of $\mathrm{NaOH}(0.1 \mathrm{M})$ were gently shaken at $37{ }^{\circ} \mathrm{C}$ for $48 \mathrm{hrs}$ to enable maximal Dox loading. The $\mathrm{pH}$ of the solution was then adjusted from basic to neutral by the addition of $0.1 \mathrm{M}$ aqueous $\mathrm{HCl}$ solution. The NP dispersion was then isolated via centrifugation (4500 rpm, $20 \mathrm{~min}$ ), washed repeatedly with water until no Dox was detected in the supernatant, and finally redispersed in water to form stable aqueous suspensions of Dox@Fl-PVP-MNPs. The percentage of Dox on NPs was determined by UV-vis spectroscopy. The absorbance of the residual Dox in the supernatant was measured $\left(\lambda_{\max }=490 \mathrm{~nm}\right)$ and the percentage of Dox loading $(w / w \%)$ was then quantified. Triplicate experiments were conducted and average values were plotted. 


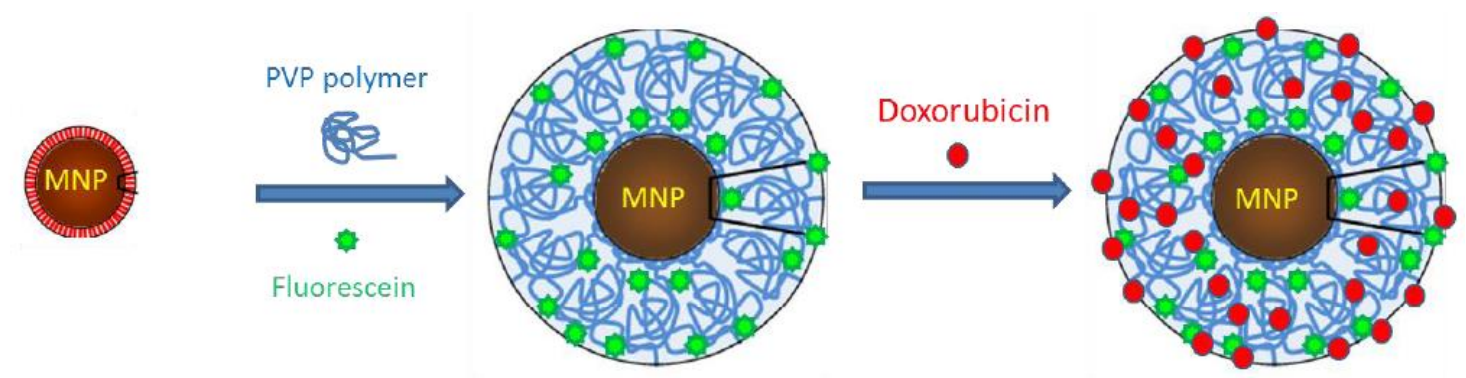

Acld-stabllized MNP

FI-PVP-MNP

Dox@FI-PVP-MNP

Fig. 1. Schematic representation for the synthesis of Dox-loaded fluorescein-labeled PVPylated magnetic nanoparticles.

Drug Release. In a typical study, a certain amount of dried drug-loaded NPs $(100 \mu \mathrm{g})$ were suspended in $1 \mathrm{~mL}$ PBS buffer at two different $\mathrm{pH}$ values $(\mathrm{pH}=7.4$ and $\mathrm{pH}=4.0)$ and gently rotated at $37^{\circ} \mathrm{C}$. The concentration of Dox released in the supernatant was determined at fixed time intervals by UV-vis spectroscopy. The drug concentration could be directly calculated from the measured absorbance. Triplicate aliquots were run for each time interval, and average values were plotted.

Prussian blue staining. Cancer cells $\left(1.5 \times 10^{4}\right.$ cells/well $)$ were allowed to attach overnight in a 6 -well plate at $37^{\circ} \mathrm{C}$ and $5 \% \mathrm{CO}_{2}$. The media was removed. MNPs at two different concentrations (20 or $50 \mu \mathrm{g} / \mathrm{ml}$ ) were then added and incubated with the cells for $30 \mathrm{~min}$, after which the supernatant was removed and the cells were washed three times with PBS. The cells were fixed with $10 \%$ formalin $(0.5$ $\mathrm{ml} /$ well) for $5 \mathrm{~min}$, and then washed with PBS twice. To each well was added $0.5 \mathrm{ml}$ of a $1: 1$ mixture of $4 \%$ potassium ferrocyanide (II) trihydrate and $4 \% \mathrm{HCl}$ solution, and the cells were incubated in the dark at $37^{\circ} \mathrm{C}$ for $30 \mathrm{~min}$. The staining solution was removed, and the cells were washed with PBS, counterstained with nuclear fast red, and imaged.

Cell viability assay. Viability of cancer cells exposed to MNPs was determined using MTT assay following the manufacturer's protocol. The cell lines were first seeded in a 96-well plate at a density of $1 \times 10^{5}$ cells/well and incubated in $5 \% \mathrm{CO}_{2}$ at $37{ }^{\circ} \mathrm{C}$. MNPs at different concentrations were then added. After $48 \mathrm{hrs}$ of incubation, the media was removed and the cells were washed with PBS. $20 \mu \mathrm{l}$ of MTT reagent $(5 \mathrm{mg} / \mathrm{mL})$ was added to each well and kept for $4 \mathrm{hrs}$ at $37^{\circ} \mathrm{C}$ in the incubator. The colored MTT formazan were dissolved in DMSO. The absorbance was measured on iMark microplate absorbance reader at $590 \mathrm{~nm}$.

\section{RESULTS AND DISCUSSION}

\section{A. Synthesis of Dox@Fl-PVP-MNPs}

The construction of Dox-loaded PVP-Fl-MNPs is illustrated in Fig. 1. Briefly, oleic acid-stabilized $\mathrm{Fe}_{3} \mathrm{O}_{4}$ MNPs were prepared using the Ko-precipitation Hydrolytic Basic $(K H B)$ approach, according to our previously published work [12]. Oleic acids chelated to the surface of $\mathrm{Fe}_{3} \mathrm{O}_{4} \mathrm{NPs}$ via the carboxylates were then exchanged with PVP in the presence of fluorescein (Fl), followed by simple Dox adsorption to furnish Dox-loaded PVP-Fl-MNPs. The non-covalent chemistry was chosen because it is easy, simple and has the advantage to preserve the structure of both MNPs and the attached Dox, thus enabling successful intracellular tracking of the drug. We hypothesized that the PVP polymer would anchor at the interface to confer an aqueous dispersity to the formulation and the amphiphilic drug would adsorb and partition into the PVP/Fl shell surrounding the $\mathrm{Fe}_{3} \mathrm{O}_{4}$ NPs. Our studies demonstrated that this simple approach formed water-dispersible drug-loaded NP formulation, with relatively high drug loading efficiencies.

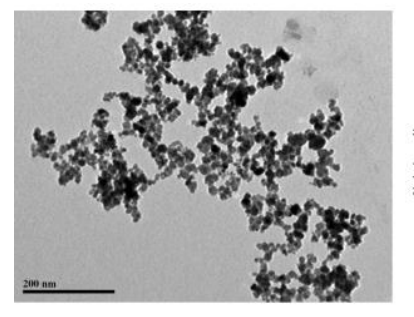

(a)

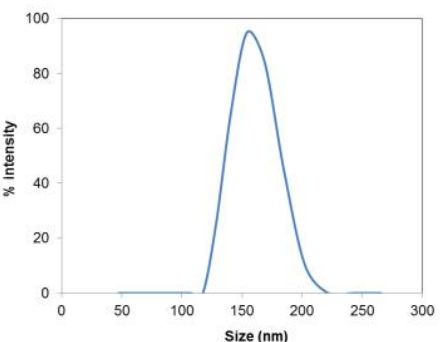

(b)
Fig. 2. TEM image of aqueous dispersion of Dox-loaded Fl-PVP-MNPs. DLS measurement of an aqueous dispersion of Dox-loaded Fl-PVP-MNPs showing hydrodynamic diameter $\left(D_{H}\right)=150 \mathrm{~nm}$.

Fig. 2(a) shows TEM images of a sample of Dox-loaded PVP-Fl-MNPs, indicating core diameter $\sim 8-10 \mathrm{~nm}$. No noticeable change to the NP features before and after Dox loading was evident from the TEM images, indicating the stability during drug loading process. DLS measurements of a dispersion of Dox@PVP-Fl-MNPs in water revealed a relatively sharp peak at $\sim 150 \mathrm{~nm}$, pinpointing the uniformity of the as-synthesized particles (Fig. 2(b)). Moreover, DLS measurements were recorded after 6 months showing no significant change in the size, confirming the stability of the particles in their aqueous dispersions. FTIR was further extended to study the conjugation of Dox to the NPs (Fig. 3). FTIR spectrum of Dox@PVP-Fl-MNPs clearly shows the characteristic peaks for pure Dox with $\mathrm{N}-\mathrm{H}, \mathrm{O}-\mathrm{H}$, anthraquinone $\mathrm{C}=\mathrm{O}$, and $\mathrm{C}-\mathrm{O}$ stretching vibrations (i.e. 3520, $3321,1617 / 1579$, and $990 \mathrm{~cm}^{-1}$ ) respectively. Peaks at 2920, $2835 \mathrm{~cm}^{-1}$ are due to PVP, Dox and Fl C-H stretching vibrations. Fe-O stretchings at $~ 560$ and $610 \mathrm{~cm}^{-1}$ confirmed the presence of iron oxide in the core of the construct.

Importantly, the spectrum showed the disappearance of the band at $1731 \mathrm{~cm}^{-1}$ corresponding to C-13 carbonyl of Dox. The N-H stretching vibrations of the primary amine at $\sim 3520$ $\mathrm{cm}^{-1}$ and the bands observed at $\sim 805 \mathrm{~cm}^{-1}$ due to the N-H wag in pure Dox seem to disappear in the FTIR spectrum of Dox@PVP-Fl-MNPs. All this indicates that the attachment of Dox to Fl-PVP-MNP construct occurs via the interaction of $-\mathrm{NH}_{2},-\mathrm{OH}$, and carbonyl groups of Dox to Fl-PVP-MNPs, 
consistent with previous reports [13]. Mild shifts in IR spectrum and lack of new peaks formed upon Dox adsorption indicate that no significant structural changes occurred to either Dox or the NP construct. TGA data showed a 50\% weight loss of the Dox@Fl-PVP-MNPs, further confirming the coating with the PVP polymer, Fl and Dox.
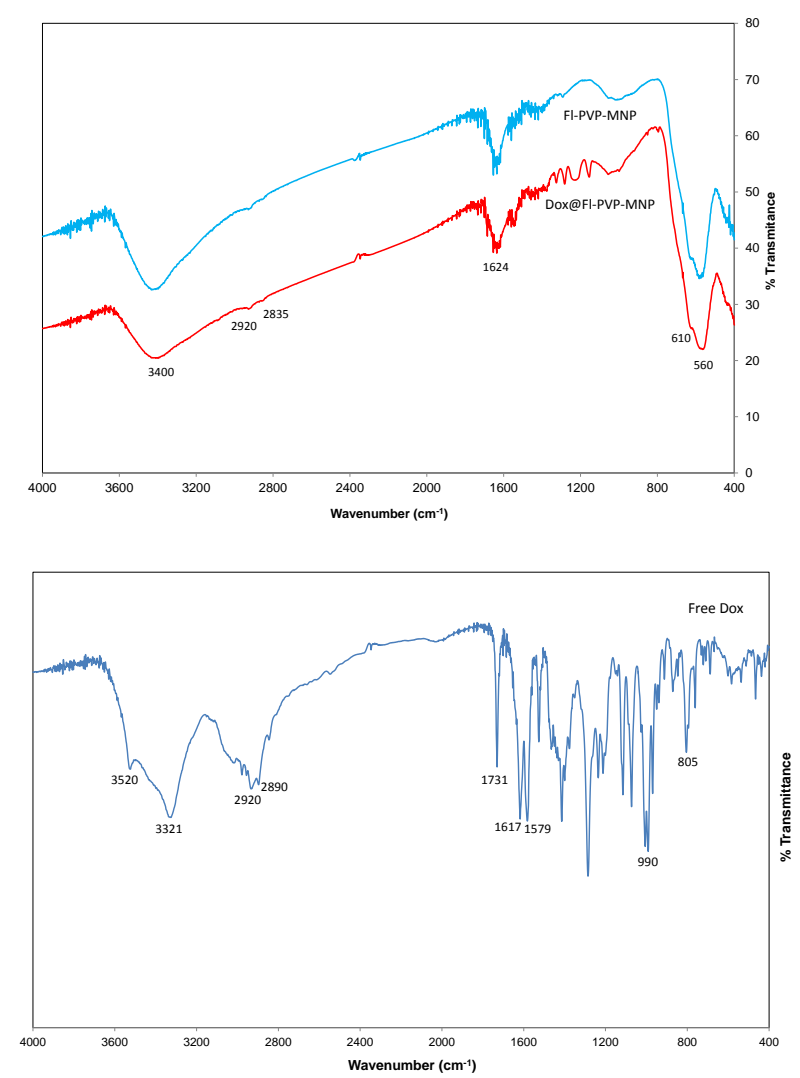

Fig. 3. FTIR spectra for Fl-PVP-MNP and Dox @Fl-PVP-MNP (left) and free Dox (right), clearly showing the peaks associated with Dox, and its successful loading.

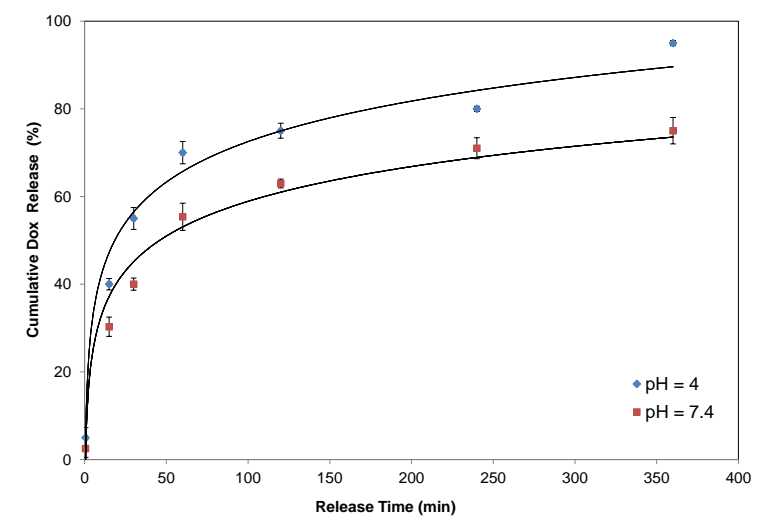

Fig. 4. Drug-release profiles for Dox-loaded Fl-PVP-MNPs measured at two different $\mathrm{pHs}$ in PBS buffer at $37^{\circ} \mathrm{C}$.

\section{B. Drug Loading and Release}

We then explored drug loading efficiencies and in vitro release rates. First, loading of Dox onto the surface of the flourscinated PVP-coated iron oxide NPs was studied. Dox consists of a weak amphipathic amine $\left(\mathrm{p} K_{\mathrm{a}}=8.25\right)$ and hydroxyl-substituted anthraquinone that can be easily protonated or deprotonated under acidic or basic conditions. It was found that up to $49 \%$ of the drug $(245 \mu \mathrm{g}$ out of $500 \mu \mathrm{g})$ was loaded onto Fl-PVP-MNPs, as evident from UV-vis measurements. By increasing the $\mathrm{pH}$ of the solution above the pKa of Dox, we deprotonate Dox during the loading process, and hence relatively good loading efficiencies were attained. It is worth pinpointing that lower loading efficiencies was attained at acidic or neutral $\mathrm{pHs}$.

Next, in vitro drug release studies of Dox@Fl-PVP-MNPs in PBS buffer was investigated at pH 7.4 and 4 at $37^{\circ} \mathrm{C}$ (Fig. 4). A fast initial drug release over the first 15 minutes at both pHs, representing $50 \%$ of Dox content at $\mathrm{pH}=7.4$ and $60 \%$ at $\mathrm{pH}=4$ was observed. This step was followed by a more controlled slow release, with almost quantitative discharge at $\mathrm{pH}=4$ as opposed to a $78 \%$ at neutral $\mathrm{pH}$ after $4 \mathrm{hrs}$. This initial fast release rate is likely due to the weakly bound Dox and the increased hydrophilicity of Dox at neutral or acidic $\mathrm{pHs}$ resulting in a rapid diffusion of the physically adsorbed drug molecules. It is hence accepted that the Dox molecules are adsorbed on the NPs via weak hydrophobic and/or electrostatic interactions and liberated via a diffusion-controlled manner in relatively short period of time. This fast release of Dox is unique and probably preferable to reach toxic concentrations of Dox in cells, achieving efficient cytotoxicities and hence sufficient dosages of the antitumor drug in the process of tumor therapy. Thus, the drug release performance of the PVPylated MNPs makes it a promising pharmaceutical carrier for imaging, drug delivery and therapeutics.

\section{Cellular Studies and Uptake}

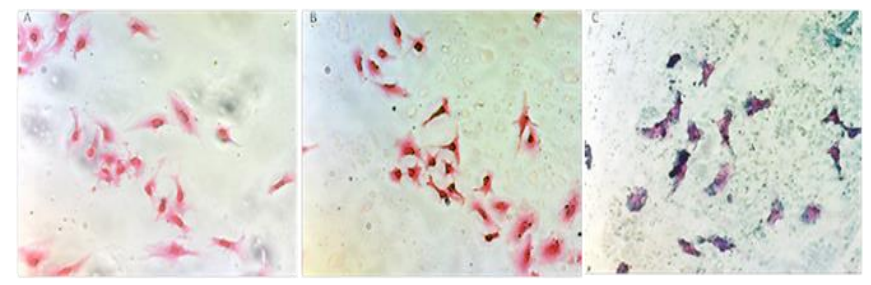

Fig. 5. Prussian blue staining images of HeLa cells incubated without or with 20 and $50 \mu \mathrm{g} / \mathrm{mL}$ of MNPs. Images were taken after 30 minutes of incubation. The blue stains clearly illustrate the successful uptake of the MNPs on the walls and inside the cytoplasm of the cells.

As an initial investigation for cellular uptake, we used Prussian blue staining, which yields a blue color upon reaction with the magnetite core, allowing easy tracking of the particles. Incubating cancerous HeLa cells with $20 \mu \mathrm{g} / \mathrm{mL}$ Fl-PVP-MNPs $(7.5 \mu \mathrm{g} \mathrm{Fe} / \mathrm{mL})$ for only 30 minutes, resulted in strong blue stains both on cell surfaces and inside the cells, suggesting a fast cellular surface binding and intracellular uptake (Fig. 5). As expected, increasing the concentration of MNPs resulted in sturdier blue color inside the cytoplasm of the cells. Next, cell viability by 3-(4,5-Dimethylthiazol-2-yl)2,5-Diphenyltetrazolium Bromide, commonly known by the MTT assay was evaluated. MTT revealed that the Fl-PVP-MNPs were not toxic to cancerous cells up to a concentration of $100 \mu \mathrm{g} / \mathrm{mL}$ of MNPs (Fig. 6). Nevertheless, Dox@Fl-PVP-MNPs were cytotoxic to HeLa cells, where killing of cells were evident even at very low doses of 10 $\mu \mathrm{g} / \mathrm{mL}$ (Fig. 6). Our results suggest that the cytotoxicity of Dox-loaded Fl-PVP-MNPs is due exclusively to delivery of Dox to cells and not to any inherent toxicity of NPs. We hypothesize that the observed cytotoxic effect from the Dox-PVP-MONPs is due to both the drug released once the 
NPs encountered the cell membrane, as well as the uptake of NPs inside the cells releasing the drug intracellularly. It is worth pinpointing that following uptake, the lower $\mathrm{pH}$ inside endosomes is expected to further facilitate the release of Dox from the NPs due to increased hydrophilicity of the drug, which can then translocate to the nucleus and exert its cytotoxic action.

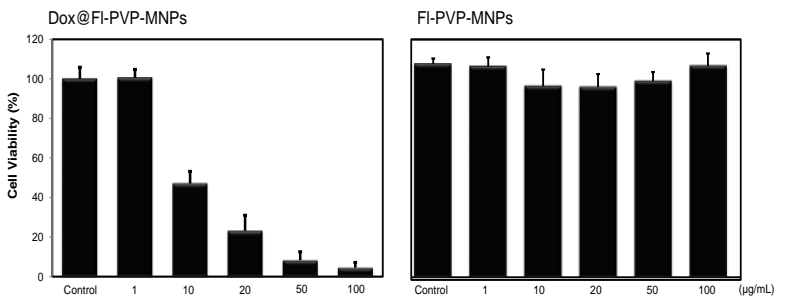

Fig. 6. MTT assay: Percent of viable cells upon incubation for $48 \mathrm{hrs}$ with different concentrations of Fl-PVP-MNPs up to $100 \mu \mathrm{g} / \mathrm{mL}$, showing no toxicity to the cancerous cells. Cells exposed to Dox-loaded Fl-PVP-MNPs show considerable decrease in cell viability even at very low doses of 10 $\mu \mathrm{g} / \mathrm{mL}$

In order to further confirm the uptake and intracellular drug delivery of Dox-loaded Fl-PVP-MNPs by cells, live imaging using confocal microscopy was employed. The acquired images confirmed that Dox is indeed delivered to the cell cytoplasm in relatively short periods of time in 1-4 hours, preferably 4 hours, and to the nucleus after $4-12$ hours, preferably 6 hours (Fig. 7). The intensity of the red fluorescence increased gradually with time, resulting from the drug release of Dox-loaded Fl-PVP-MNPs. Importantly, this delivered payload is promising to enhance the effectiveness of the drug in cancer patients and may further allow clinicians to image the tumor cells exposed to the MNPs.

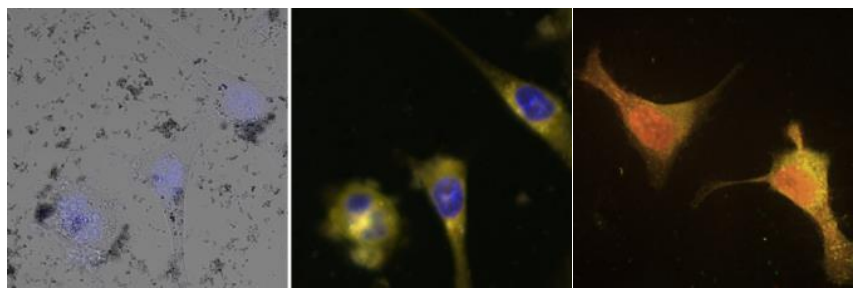

Fig. 7. Live confocal microscopy images of cancerous cells incubated with Dox@Fl-PVP-MNP (10 $\mu \mathrm{g} / \mathrm{mL}$ NPs) after 4 and 18 hrs. (a) Transmitted light; (b) Overlay (yellow) of Dox channel (red), (b) Fluorescein channel (green), (c) Hoechst channel (blue). Blue color shows the location of the nuclei. The images clearly show the internalization of NPs inside the cell cytoplasm after $4 \mathrm{hrs}$, and effective delivery of Dox to the nucleus after overnight treatment.

\section{CONCLUSION}

In conclusion, we embarked on a simple and practical approach to prepare novel formulation of magnetic fluorescent NPs loaded with anticancer drugs with high loading efficiencies. While the Fl-PVP-MNPs were highly biocompatible and not toxic to cells, the Dox@Fl-PVP-MNPs was found to be much more potent to cancerous cells, causing killing to the cells at relatively low doses. The Dox@Fl-PVP-MNPs were found to be uptaken intracellualry by the cells, where Dox is then released and translocated to the nucleus exerting its cytotoxic action, in relatively short periods of time. Importantly, the Dox-loaded PVPylated MNPs described here can potentially open new opportunities for in vivo therapeutic imaging, cancer monitoring, and hyperthermia.

\section{ACKNOWLEDGMENT}

This work was funded by KAIMRC (RC13/204/R). The author acknowledges KSAU-HS and KAIMRC for their continuous support. Special thanks to M. Alghamdi and A. Rizwan for their help in the characterization of the MNPs and the cellular studies, respectively.

\section{REFERENCES}

[1] S. Mura, J. Nicolas, and P. Couvreur, "Stimuli-responsive nanocarriers for drug delivery," Nat Mater, vol. 12, pp. 991-1003, 2013.

[2] G. Tiwari, R. Tiwari et al., "Drug delivery systems: An updated review," Int. J. Pharm. Investig., 2012.

[3] R. K. Jain and T. Stylianopoulos, "Delivering nanomedicine to solid tumors," Nat Rev Clin Oncol, vol. 7, pp. 653-664, 2010.

[4] L. Zhang, "Nanoparticles in medicine: Therapeutic applications and developments," Clin Pharmacol Ther, vol. 83, pp. 761-9, 2008.

[5] V. P. Torchilin, "Targeted pharmaceutical nanocarriers for cancer therapy and imaging," AAPS J, vol. 9, 2007.

[6] V. Wagner, A. Dullaart, A. K. Bock, and A. Zweck, "The emerging nanomedicine landscape," Nat Biotechnol, vol. 24, 1211-1217, 2006.

[7] M. H. El-Dakdouki, D. C. Zhu et al., "Development of multifunctional hyaluronan-coated nanoparticles for imaging and drug delivery to cancer cells," Biomacromolecules, vol. 13, pp. 1144-1151, 2012.

[8] T. K. Jain, J. Richey et al., "Magnetic nanoparticles with dual functional properties: Drug delivery and magnetic resonance imaging," Biomaterials, vol. 29, pp. 4012-4021, 2008.

[9] J. Dobson, "Magnetic nanoparticles for drug delivery," Drug Dev Res, vol. 67, pp. 55-60, 2006.

[10] C. Sun, J. S. H. Lee, and M. Zhang, "Magnetic nanoparticles in MR imaging and drug delivery," Adv Drug Deliv Rev, vol. 60, pp. 1252-1265, 2008.

[11] H. K. Sajja, M. P. East, H. Mao, Y. A. Wang, S. Nie, and L. Yang, "Development of multifunctional nanoparticles for targeted drug delivery and noninvasive imaging of therapeutic effect," Curr Drug Discovery Technol, vol. 6, pp. 43-51, 2009.

[12] K. El-Boubbou, R. O. Al-Kaysi et al., "Ultra-small fatty acid-stabilized magnetite nanocolloids synthesized by in situ hydrolytic precipitation," Journal of Nanomaterials, p. 11, 2015.

[13] S. Kayal and R. V. Ramanujan, "Doxorubicin loaded PVA coated iron oxide nanoparticles for targeted drug delivery," Mater Sci Eng C, vol. 30, pp. 484-490, 2010.

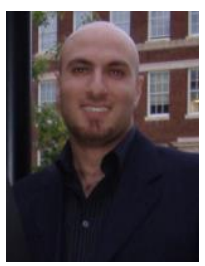

K. El-Boubbou received his B.Sc. degree in chemistry from Lebanese University (2003), and M.Sc. from American University of Beirut (2005) working in the field of physical organic chemistry. In 2010, he received his Ph.D. in chemistry from Michigan State University. His work centered on the development of carbohydrate-functionalized magnetic iron oxide nanoparticles for detection of bacteria, cancer and atherosclerosis. In 2007, he received an American Heart Association (AHA) predoctoral fellowship award where he worked on the synthesis of biocompatible targeted contrast agents for the molecular imaging and detection of atherosclerosis. His findings in this regard sets the stage for the lab that utilized those nanoparticles for various applications including macrophage targeting and drug delivery to cancer cells and tumors, to name few. In 2010, he was elected as the recipient of Alberta Ingenuity Centre for Carbohydrate Science (AICCS) Young Investigator Award. He started his postdoctoral career at the University of Vermont working on functional mesoporous silica materials as encapsulation and delivery devices for chemical and biological applications. In 2013, Dr. El-Boubbou joined King Saud bin Abdulaziz University for Health Sciences (KSAU-HS) as an Assistant Professor of Chemistry. His research focuses on the design of functional nanoscale materials that could be utilized for various biological and biomedical applications, specifically synthesis of metal oxide nanoparticles for imaging, delivery and theranostics. Dr. El-Boubbou is a member of RSC, ACS, AHA and serves as referee and reviewer for various publishers. 